\title{
Le syndrome des mangeurs de fromage
}

II existe un «syndrome des buveurs de laít» ou syndrome de Burnett; il s'agit, rappelons-le, de lithiase rénale ou de néphrocal-cinose qui se produit chez des ulcéreux buvant chaque jour un ou plusieurs litres de lait; celui-ci fournit le calcium qui précipite dans les bassinets des reins. En réalité, ces ulcéreux étaient en même temps - et surtout - des gros consommateurs d'alcalins (comme dans la deplorable diète de Sippy) responsables d'une alcalinisation des urines qui empêche la solubilisation des sels de chaux. D'où le nom anglais plus explicite de « milk-alkalisyndrome $»$; le lait fournit bien la matière premiere de la lithiase, mais seraít incapable, à luí seul, de la provoquer s'il n'y avait pas des excès de bicarbonates.

On nous annonce maintenant d'Angleterre un «cheese syndrome». Un médecin de Sheffield, S. S. Maan, à propos de malaises ressentis par une malade de 26 ans à la suite d'un traitement au Parnate puis à la Parstelín, note curieusement dans une «correspondance» du Lancet $(21 \mathrm{sept}$. 1963, p. 639): «She relished cheese and ate large quantities at any time of the day.» Peu après, B. Blackwell etablissait la relation de cause à effet entre Гíntolérance aux deux medicaments et Tíngestion de fromage, et de nombreuses observations cliniques ont suivi, complétées par des recherches expérimentales.

Le principe actif du Parnate et de la Parstelin est la tranylcypro-míne, un ínhibíteur de la monoamíne-oxydase (MAO). La liste est longue des medicaments de ce type, utilises avant tout comme anti-dépressifs et également contre $\Gamma$ angor pectoris. Parmi ceux commercialises en Suisse, retenons Tiproniazide (Marsílíd), Гisocarboxazide (Marplan), la nialamide (Niamid), la phénelzine (Stírneval) et la pivhydrazine (Tervasid), qui font partie des thymo-analeptiques, à effet essentiellement euphorisant et stimulant; la tranylcypromine est classée dans ce groupe. Sur le plan clinique, une publication de A. J. Cooper et coll., de Bristol (Lancet du 7 mars 1964, p. 527), analyse les reactions d'into-lérance de 137 malades traités à la Parstelin; 27 d'entre eux, soit 20\%, firent des crises sévères d'hypertension, avec céphalées intenses, raideur de la nuque, photophobie, palpitations, nausées et vomisse-ments, et jusqu'à quelques hémorragies cérébrales fatales. Or dix de ces accidents survinrent chez des sujets quí avaient mange du fromage une à troís heures auparavant!

Editorial

183

On sait depuis le debut de ce siècle que les bactéries cultivées dans un hydrolysat de caséine engendrent de la tyramine à partir de la tyrosine; on en trouve $0,06 \%$ dans le fromage d'Emmenthal et jusqu'à 0,2\% dans le Camembert. La tyramine est un sympathicomimétique

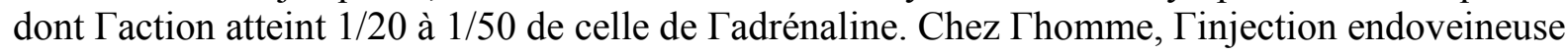
ou sous-cutanée de 20 à $80 \mathrm{mg}$ de tyramine produit une hypertension marquee. Cette amine est normalement dégradée, sous $\Gamma$ influence d'une MAO et de Гaldéhyde-oxydase en acide phydroxy-phényl-acétique, non toxique.

A. M. Aasator et coll. ont pu démontrer (Lancet du 5 oct. 1963, p. 733) que l'ingestion par un adulte sain de $180 \mathrm{~g}$ de Camembert ou $225 \mathrm{~g}$ de fromage de Stilton provoquait une augmentation 
respective de $200 \%$ et $70 \%$ des acides phénoliques dans les urines, acides phéno-liques provenant presque entièrement de Гoxydation de la tyramine par la MAO.

Plus récemment, B. Blackwell et E. Marley (Lancet du 7 mars 1964, p. 530) ont repris le problème chez le chien et le rat et montrent que la substance nocive contenue dans les fromages agit sur les récepteurs adrénergiques, mais n'est pas une cathécolamine. L'effet toxique se manifeste après une seule dose et n'est pas la consequence d'une elevation du taux dans les tissus à la suite d'une administration prolon-gée ou répétée.

Le mêcanísme de $\Gamma$ intoxication serait alors le suivant: la tyramine contenue dans le fromage, et qui doit être dégradée par la MAO du foie, pénètre dans la circulation quand des inhibiteurs de la MAO empêchent $\Gamma$ action protectrice de ce ferment; la tyramine exerce alors son eífet hypertensif sur Гorganisme. Si de pareils accidents n'ont pas été décrits en Europe continentale, cela tient avant tout à la puissance de la tranylcypromine, qui est 40 fois plus forte que Гiproniazid, par exemple.

Aussi, sur la recommandation de la Food and Drug Administration américaine, les preparations comme le Parnate et la Parstelin ont-elles été retirees du marché aux U.S.A. Et d'autre part, on avertit systéma-tiquement les malades recevant des inhibiteurs de la MAO, d'éviter la consommation de fromage à dose élevée. Cette precaution reste valable pour les sujets traités avec des inhibiteurs moins violents de la MAO, mais dont le foie est insuffisant: l'addition de la drogue jointe à Гincapacité partielle du foie (lieu où les MAO dégradent la tyramine) pourraient produire le même syndrome hypertensif. C'est une piste à

184

Editorial

explorer chez les sujets qui prétendent ne pas supporter les fromages, quand des facteurs non digestifs n'interviennent pas.

Voici done un nouvel exemple de maladie iatrogène, au second degré si l'on peut dire; ici le medicament produit les sígnes graves d"'intolerance par Гintermêdiaíre d-'un aliment, inoffensif $\mathbf{s}^{1} / 81$ en est: le lait pour le syndrome de Burnett, le fromage pour le «Cheese syndrome». Ce ne sera pas fait pour faciliter la diététique hospitalière. . .

M.D. 